\title{
Onychomycosis due to mixed infection with non- dermatophyte molds and yeasts
}

\section{Funda Tamer ${ }^{1}$, Mehmet Eren Yuksel ${ }^{2}$}

${ }^{1}$ Ufuk University School of Medicine, Department of Dermatology, Ankara, Turkey, ${ }^{2}$ Aksaray University School of Medicine, Department of General Surgery, Aksaray, Turkey

Corresponding author: Dr. Funda Tamer, E-mail: fundatmr@yahoo.com

\begin{abstract}
Onychomycosis is the fungal infection of nails which affects $5.5 \%$ of the general population. Etiologic agents include dermatophytes, non-dermatophyte molds, and yeasts. The infection usually occurs due to dermatophytes. However, non-dermatophyte molds and yeasts have an increasing role in the development of onychomycosis. Detecting causative agent is crucial for the appropriate therapy, as non-dermatophytic molds and yeasts are usually resistant to classical antifungal agents which are used in the treatment of onychomycosis. Hereby, we report a 39-year-old Caucasian male patient with onychomycosis of the great toenails caused by Aspergillus niger complex, Chaetomium globosum, Cladosporium species, Candida species, and onychomycosis of the left thumbnail due to Aspergillus niger, Chaetomium globosum, Cladosporium species and Candida lambica.
\end{abstract}

Key words: Nail fungus; Non-dermatophyte molds; Onychomycosis; Yeasts

\section{INTRODUCTION}

Onychomycosis is the fungal infection of nails caused by dermatophytes, non-dermatophyte molds, and yeasts. Onychomycosis is the most common nail disease with the prevalence of $5.5 \%$ all over the world [1]. It usually affects toenails of adults [2]. Onychomycosis can lead to pain, paresthesia, difficulties in daily activities, impaired social interactions, and low self-esteem [1,3]. Trauma, tinea pedis, advanced age, diabetes, psoriasis, malignancy and immunosuppression are regarded as risk factors in the etiology of onychomycosis [1].

Dermatophytes, especially T. rubrum and T. mentagrophytes are regarded as the most common causative agents in onychomycosis. Nondermatophyte molds including Scopulariopsis brevicaulis, Aspergillus spp, Acremonium, Fusarium spp, Alternaria alternate, and Neoscytalidium are detected in approximately $20 \%$ of the patients. Yeasts (Candida spp.) are responsible for 10\%-20\% of cases with onychomycosis [1]. Mixed infections with dermatophytes and non-dermatophyte molds in onychomycosis have been rarely reported [4].

However, an increasing role of non-dermatophyte molds and yeasts in onychomycosis has been described [5,6]. Furthermore, it has been suggested that nondermatophytic molds and yeasts are significantly more prevalent causative agents in onychomycosis than dermatophytes [6]. Ovcina-Kurtovic reported Candida albicans as the most common fungus isolated from psoriatic patients with nail involvement [7].

Antifungal drug resistance is becoming a healthcare problem as a result of their wide use and availability [8]. Patients with onychomycosis due to non-dermatophytic molds or yeasts may not respond to systemic antifungals like itraconazole, fluconazole and griseofulvin which are frequently recommended in the treatment of onychomycosis [5]. Identification of the causative agent with mycological examination is needed for the appropriate treatment and good clinical outcome [6].

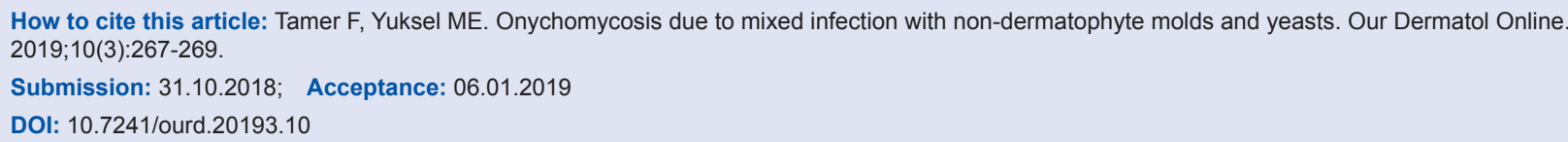




\section{CASE REPORT}

A 39-year-old Caucasian male patient presented with a 5-year history of discoloration and thickening of the toenails. The patient stated that the symptoms started as a yellow discoloration and thickening under the tip of the right great toenail. Then it spread to the rest of his toenails and left thumbnail gradually. The patient had pain and tenderness in the great toenails while wearing shoes. He did not receive any medication previously. The past medical history was remarkable for chronic peripheral venous insufficiency. He has been wearing compression stockings for the last two years. The family history was unremarkable. The patient denied nail injury, getting pedicure at a nail salon, walking barefoot in public areas like swimming pool, sauna or using an immunosuppressive agent.

Physical examination revealed a squamous plaque on the extensor surface of the left thumb; hyperkeratotic, yellow groove in the midline of the left thumbnail extending from proximal nail fold to distal edge; onycholysis, and white, opaque, friable lesions which created a linear plaque on the left thumbnail (Fig. 1). Moreover, subungual hyperkeratosis, yellow discoloration and onycholysis were observed in all toenails (Figs. 2-4).

The laboratory tests including complete blood count, fasting blood glucose, creatinine, total cholesterol, triglyceride, alanine aminotransferase, aspartate aminotransferase, ferritin, folate, vitamin B12, 25-hydroxyvitamin $\mathrm{D}$, zinc and thyroid stimulating hormone levels were all within normal limits.

Mycological examination was performed using conventional methods and matrix assisted laser desorption ionization time of flight mass spectrometry (MALDI-TOF MS). The specimens were obtained from scrapings of left thumbnail and from both great toenails. Aspergillus niger complex, Chaetomium globosum, Cladosporium species and Candida species were identified from the scrapings of bilateral great toenails. Aspergillus niger, Chaetomium globosum, Cladosporium sp. and Candida lambica were detected from the specimens obtained from the left thumbnail. Thus, the diagnosis of onychomycosis due to mixed infection with non-dermatophyte molds and yeasts was made based on clinical findings and MALDITOF MS technique.

The patient was advised to get a skin biopsy from the squamous plaque on the left thumb to rule out psoriasis. However, the patient refused the biopsy. He

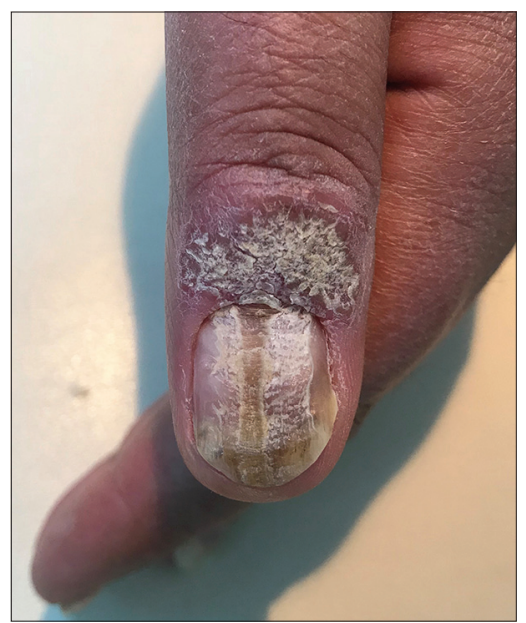

Figure 1: Onycholysis, white, friable lesions, and hyperkeratotic, yellow groove of the left thumbnail. Aspergillus niger, Chaetomium globosum, Cladosporium sp. and Candida lambica were detected from the specimens obtained from the left thumbnail.

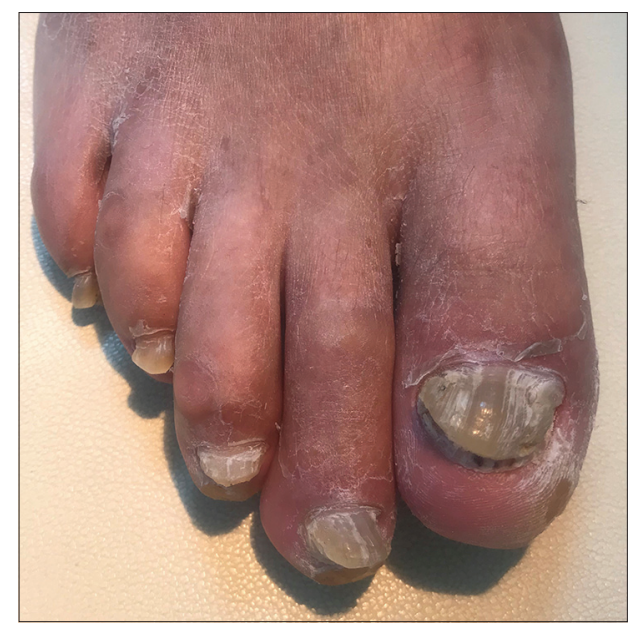

Figure 2: Subungual hyperkeratosis, yellow discoloration and onycholysis of the toenails.

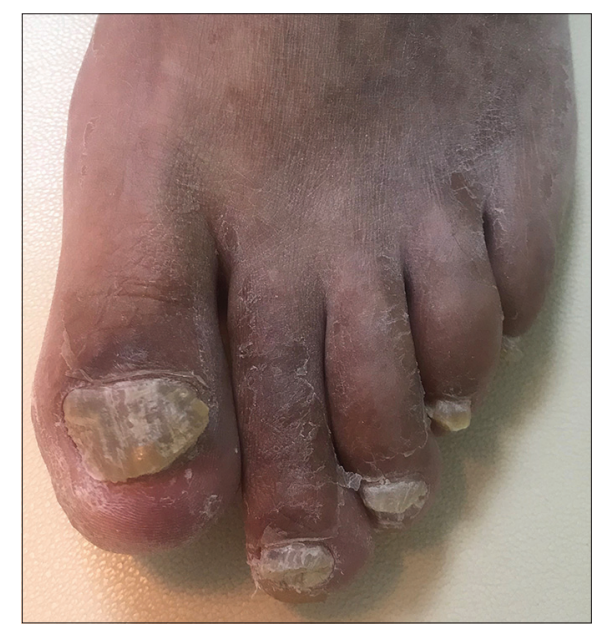

Figure 3: Total dystrophic onychomycosis of the toenails. 


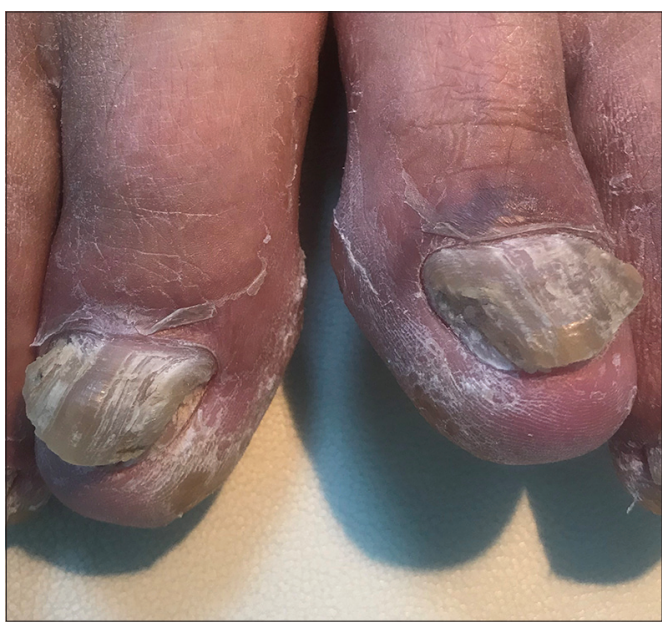

Figure 4: Closer view of the great toenails. Aspergillus niger complex, Chaetomium globosum, Cladosporium species and Candida species were identified from the scrapings of great toenails.

claimed that the lesion occured as a result of repetitive picking of the skin due to psychological stress.

\section{DISCUSSION}

Onychomycosis can clinically present with subungual hyperkeratosis, onycholysis, melanonychia, and brown, yellow, orange or white discoloration of the nail plate and friable nails. Onychomycosis due to non-dermatophytes has been associated with a marked periungual inflammation [9]. The non-dermatophyte molds including Scopulariopsis brevicaulis, Aspergillus spp., Fusarium spp., Acremonium spp., Alternaria spp. and Neoscytalidium spp. may be the primary pathogens in the development of onychomycosis. Moreover, they may play role as contaminant agents and secondary pathogens. Yeasts like Candida albicans and Candida parapsilosis cause nail infections only in patients with predisposing factors such as immunosuppression and diabetes [9].

Clinical diagnosis should be confirmed with a mycological investigation, since the treatment plan depends on the species of fungi and number of affected nails [9]. Treatment of patients with nail infections caused by non-dermatophyte organisms like Fusarium is usually difficult [10].

Treatment options include systemic and topical antifungal agents, chemical or surgical removal of the infected nail, and laser therapy. Oral antifungals may have side effects such as liver damage, and they may cause unwanted drug interactions especially in elderly. Recurrence rate of fungal nail infection is high especially in immunocompromised patients. In addition, diabetes and genetic predisposition to onychomycosis increase the rate of recurrence [10].

Hereby, we report a patient with onychomycosis of the left thumbnail and toenails due to mixed infection of non-dermatophyte molds and yeasts including Chaetomium globosum, Cladosporium species which are rare in onychomycosis etiology. Detecting the causative agent is crucial for the appropriate therapy, as nondermatophytic molds and yeasts are usually resistant to classical antifungal agents.

\section{Consent}

The examination of the patient was conducted according to the Declaration of Helsinki principles.

\section{REFERENCES}

1. Lipner SR, Scher RK. Part I: Onychomycosis: clinical overview and diagnosis. J Am Acad Dermatol. 2018 Jun 27 (Epub ahead of print).

2. Bonifaz A, Vázquez-González D, Saúl A, Fierro-Arias L, PonceOlivera MR. Refractory onychomycosis due to trichophyton rubrum: combination therapy with itraconazole and terbinafine. Our Dermatol Online. 2011;2:108-12.

3. Kaur T, Puri N. Onychomycosis - a clinical and mycological study of 75 cases. Our Dermatol Online. 2012;3:172-7.

4. Gupta AK, Nakrieko KA. Molecular determination of mixed infections of dermatophytes and nondermatophyte molds in individuals with onychomycosis. J Am Podiatr Med Assoc. 2014;104:330-6.

5. Ding CH, Rahman MM, Tzar MN, Yusoff H, Satim H. Nondermatophytic moulds and yeasts as agents of onychomycosis in a Malaysian medical centre. Bangladesh J Med Sci. 2017;16:380-3.

6. Kaur R, Panda PS, Khan S. Non dermatophytic molds causing onychomycosis: a rising trend in North India. Int J Community Med Public Health. 2017;4:4532-7.

7. Ovcina-Kurtovic N, Kasumagic-Halilovic E. Prevalence of nail abnormalities in patients with psoriasis. Our Dermatol Online. 2013;4:272-4.

8. Nigam PK. Antifungal drugs and resistance: current concepts. Our Dermatol Online. 2015;6:212-21.

9. Piraccini BM, Alessandrini A. Onychomycosis: a review. J Fungi (Basel). 2015;1:30-43.

10. Ghannoum M, Isham N. Fungal nail infections (onychomycosis): a never-ending story? PLoS Pathog. 2014;10:e1004105.

Copyright by Funda Tamer, et al. This is an open-access article distributed under the terms of the Creative Commons Attribution License, which permits unrestricted use, distribution, and reproduction in any medium, provided the original author and source are credited.

Source of Support: Nil, Conflict of Interest: None declared. 